\title{
Performance and simulation of the surface detector array of the TAx4 experiment
}

\section{Kozo Fujisue ${ }^{a, *}$ on behalf of the Telescope Array Collaboration}

(a complete list of authors can be found at the end of the proceedings)

${ }^{a}$ Instiyute for Cosmic Ray Research,

Kashiwa-no-ha 5-1-5, Kashiwa City, Japan

E-mail: fujisue@icrr.u-tokyo.ac.jp

The TAx 4 experiment is a project to observe highest energy cosmic rays by expanding the detection area of the Telescope Array (TA) experiment with newly constructed surface detectors (SDs) and fluorescence detectors (FDs). New SDs are arranged in a square grid with $2.08 \mathrm{~km}$ spacing at the north east and south east of the TA SD array. We use CORSIKA simulations and implement the calibration data of the new SDs to calculate the performance of the new SDs. We compare the data with the simulation and validate the performance of the SDs. The comparison and the performance will be shown in the presentation.

$37^{\text {th }}$ International Cosmic Ray Conference (ICRC 2021)

July 12th-23rd, 2021

Online - Berlin, Germany

\footnotetext{
*Presenter
} 


\section{Introduction}

TA [1] found evidence for a cluster of arrival directions of cosmic rays with $\mathrm{E}>57 \mathrm{EeV}$ (hotspot) [2]. To confirm this hotspot including subsequent TA anisotropy results [3, 4], we planned to quadruple the TA to accelerate the data collection speed. The current TAx4 SD array [5] consists of 257 plastic scintillation detectors, deployed on a square grid with $2.08 \mathrm{~km}$ spacing, covering an area of approximately 2.5 times TA SD [6] including TA SD. The basic design of the TAx4 SD is the same as that of the TA SD. Each surface detector consists of two layers of plastic scintillators with PMTs, electronics package consisting of CPU for slow signal processing, CPLD for board control, $50 \mathrm{MHz}$ sampling FADCs, FPGA for fast signal processing, GPS system, ADCs for monitoring, DACs for setting HV of PMTs, and solar power system with charge controller. The host electronics at the corresponding communication tower collects data stored at each SD using $2.4 \mathrm{GHz}$ wireless LAN communication. Figure 1 shows the TAx4 SD layout.

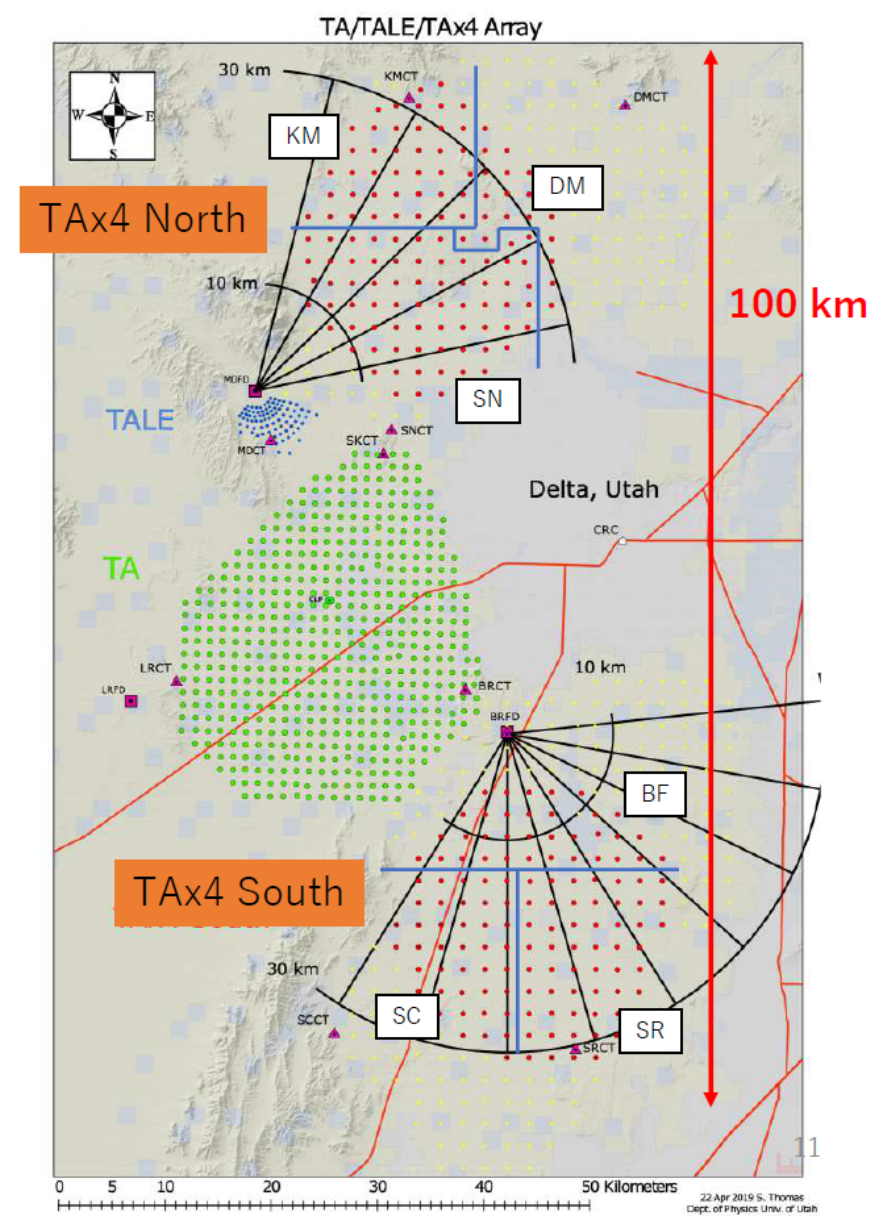

Figure 1: Layout of the TAx4. The red circles denote deployed TAx4 SDs. The yellow dots denote planned TAx4 SDs. The purple triangles denote the communication towers. The TAx4 SD array is divided into six sub arrays (KM, DM, SN, BF, SC and SR). The blue lines show the boundaries of TAx4 SD sub-arrays. The green circles denote the TA SDs. The blue circles denote the TALE SDs. The purple squares denote the fluorescence telescope stations. 
Figure 2 shows the photograph of the TAx4 SD deployed in the field.

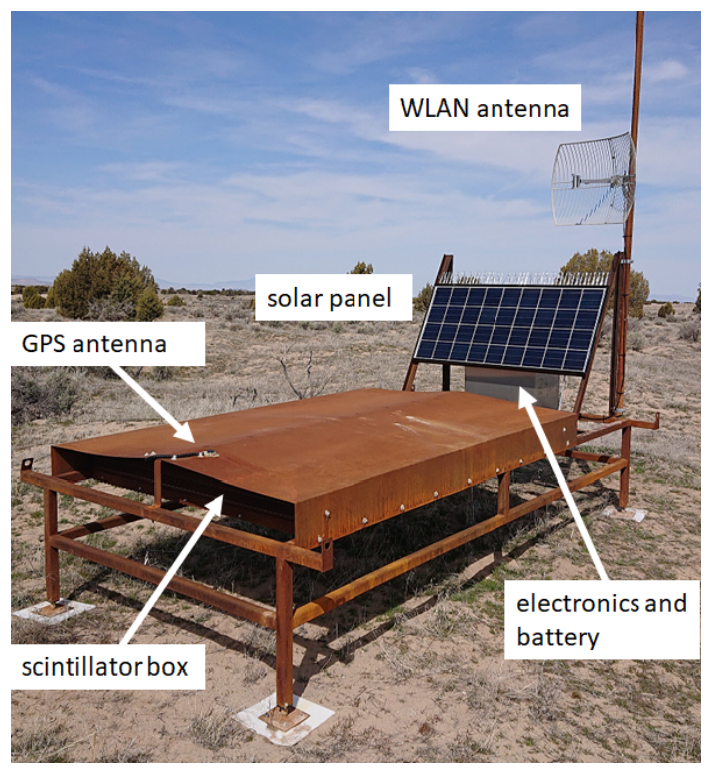

Figure 2: Photograph of the TAx4 SD deployed in the field. An SD electronics and a battery are installed in the box under the solar panel, and the scintillator box is mounted on the platform under the roof. A GPS antenna is attached on the roof. A wireless LAN antenna is attached to a pole.

\section{Trigger/DAQ and Monitor}

When both PMT signals of a surface detector exceed 0.3 MIP threshold (level-0 trigger), waveform information is recorded locally in a buffer with a time stamp by a GPS. When signals exceed 3 MIP threshold (level-1 trigger), the level-1 trigger timing information is locally stored in a trigger table, which is transmitted at $\mathrm{Hz}$ to the host electronics at the corresponding communication tower. The host electronics decides the final coincidence trigger based on the trigger tables from SDs in the sub-array with the requirement of three adjacent SD hits and a $14-\mu$ s time window (level-2 trigger) to collect an air shower event. For comparison, TA SD uses 8- $\mu$ s time window. Finally, waveform data from SDs are transmitted to the host electronics.

We have a real-time monitor system that works in the background for SD calibration and maintenance. We have the following monitors from each detector:

(every second)

(A) No. of events with > 3 MIP coincidence (for the level-1 trigger rate)

(B) GPS time stamp (GPS status)

(C) Max. clock count between 1 PPS

(every minute)

(D) No. of events with > 0.3 MIP coincidence (for the level-0 trigger rate)

(E) Battery voltage, current 
(F) Voltage of solar panel

(G) Temperature at several positions and humidity inside the detector

(every 10 minutes)

(H) Charge histogram (for gain calibration)

(I) Pulse height histogram (for linearity check)

(J) Charge histogram (for linearity check)

(K) Pedestal histogram

(L) GPS condition flag, No. of detected satellites, the condition of GPS antenna connection

Figure 3 is an example of the time variations of monitor values of one detector in the period between February 14, 2020 00:00:00 and February 22, 2020 00:00:00 in UTC.

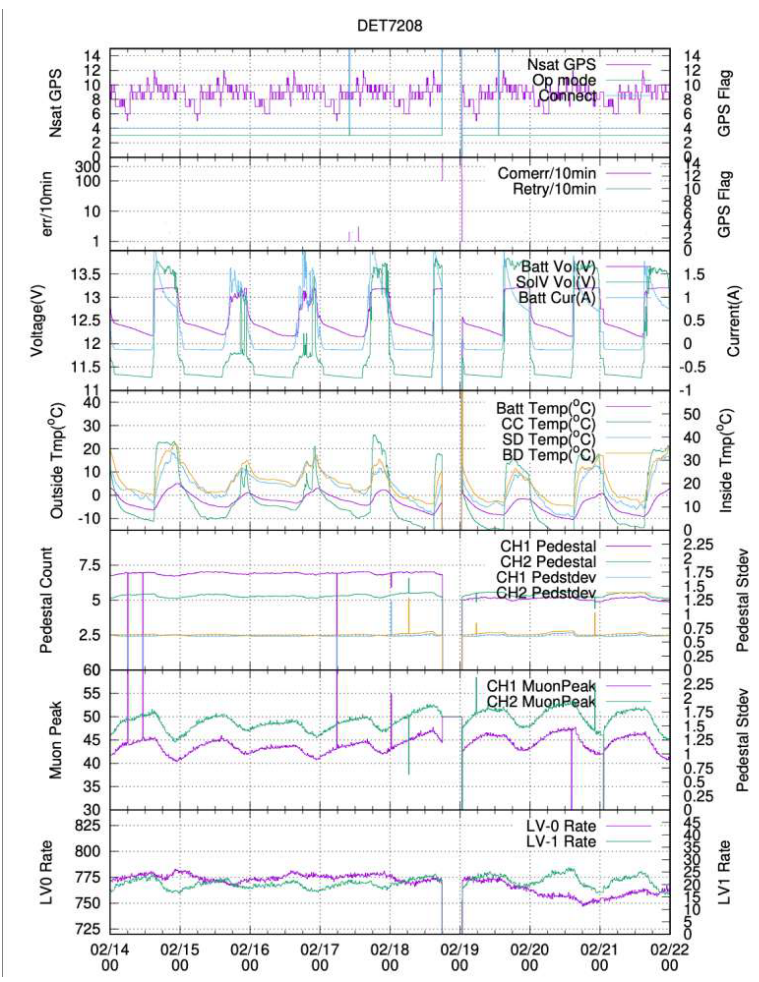

Figure 3: An example of time variations in the number of GPS satellites indicated in purple (top panel), GPS error flags (second panel), battery voltage, solar voltage and charging current indicated in purple, green and blue respectively (second panel), temperatures at a battery, charge controller, and SD box indicated in purple, green, blue respectively (third panel), pedestal average and RMS values for channel 1 in purple and green, for channel 2 in blue and yellow respectively (fourth panel), muon peaks for channel 1 in purple and channel 2 in green (fifth panel), local level-0 and level-1 trigger rates indicated in purple and green (sixth panel) for one surface detector (DET\#7208), 


\section{MC simulation}

We use an analysis method same as the TA SD with Monte Carlo (MC) simulation [7] to understand the performance of the TAx4 SD, and check the quality of the real data. We use a library of showers generated by the CORSIKA program [8] using QGSJETII-04 to model high energy hadronic interactions [9] ${ }^{1}$, FLUKA to model low energy hadronic interactions [10], an EGS4 to model electromagnetic interactions [11]. We use a dethinning algorithm [12] that enables us to reconstruct the information lost using the CORSIKA thinning option in the same way as the MC simulation of the TA SD. For this study, proton showers were used.

\section{(STEP1)}

A shower library was created with 6200 dethinning CORSIKA showers with primary energies distributed in $\Delta \log _{10} E=0.1$ bins between $10^{17.5}$ and $10^{20.5} \mathrm{eV}$. The number of showers in each bin is 200. These showers are simulated with zenith angles $(\theta)$ from $0^{\circ}$ to $60^{\circ}$ assuming an isotropic distribution ( $\sin \theta \cos \theta$ distribution at the detector level).

\section{(STEP2)}

For each simulated event, all shower particles that strike the ground are divided spatially by their landing spots into $6 \times 6-\mathrm{m}^{2}$ "tiles" on the ground and into 20 -ns wide bins by their arrival time. The total energy deposited by all particles that arrived in a particular tile, and into a virtual TAx4 SD counter located at its center, is calculated using the GEANT4 simulation package [13]. The energy deposited as a function of time is stored in the shower library.

\section{(STEP3)}

In the final step of the shower library generation, each titled shower is sampled 600 times through a detailed simulation of the detector, including Poisson fluctuation of energy deposit in the detector and electronics response and so on. The shower core positions, the azimuthal angles of the shower axis, and event times are varied in this process. The detector simulation utilizes real time calibration data from the TAx4 SD.

\section{Reconstruction}

Two fits are used to reconstruct the properties of the cosmic rays measured with the TAx4 SD in the same way as the TA SD.

\section{(Geometry fit)}

The fit to the times when counters were struck, using the modified Linsley shower shape function, is made to determine the arrival direction and the core position of the event.

\footnotetext{
${ }^{1}$ For the TA SD MC, QGSJETII-03 is used.
} 


\section{(Energy estimation)}

The primary energy estimation of events is established by measuring the charge density at 800 meters in lateral distance from the shower axis (S800). The measured particle densities from the detectors are fit to the modified AGASA lateral distribution function (LDF). The energy is firstly estimated from the table of S800 vs. $\sec \theta$ for the true MC. For the TA SD, the energies obtained from the energy estimation table are calibrated against the TA fluorescence detector using events that are observed simultaneously by both TA SD and FD. Comparing the energy scale 1.27 of the TA SD MC using QGSJETII-03 hadronic interaction model, we use the energy scale factor of 1.2 for the final energy estimation from the TAx4 SD MC using QGSJETII-04 hadronic interaction model here in this conference.

\section{1 (Performance of TAx4 SD from MC)}

We obtained $27 \%$ energy resolution and 2.4 degree angular resolution of cosmic rays with $\mathrm{E} \geq$ $57 \mathrm{EeV}$ under the conditions described in Section 5.

\section{(Example of the reconstruction of a real data)}

Figure 4 shows an example of the reconstruction of a real data that was recorded at May D9, 2020 00:36:56 in UTC.
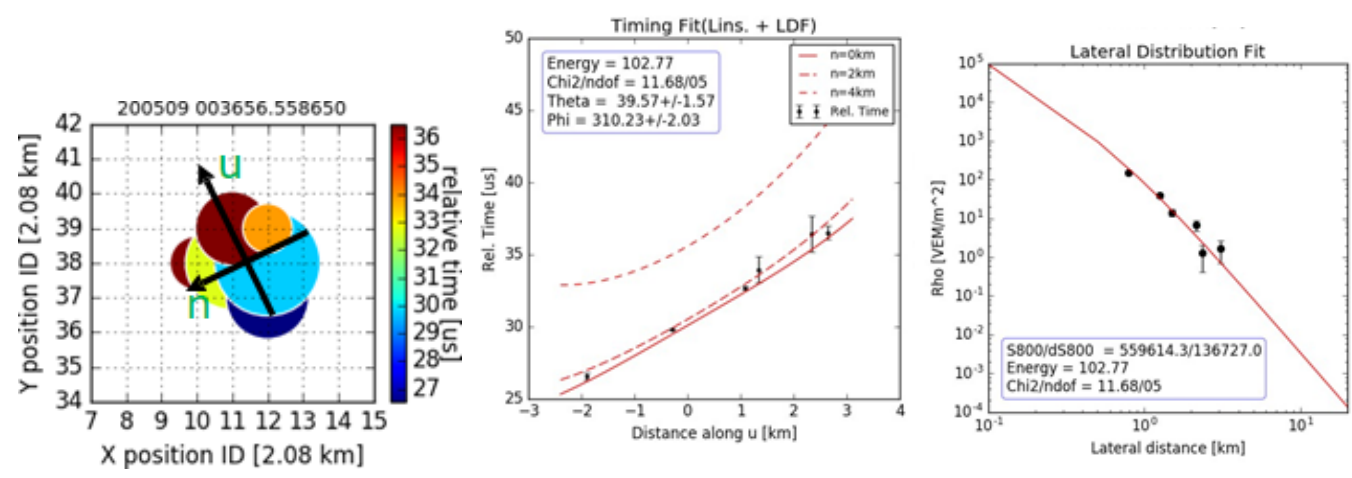

Figure 4: Example of the reconstruction of a typical TAx4 SD real data. a) Event display picture. Circles centers correspond to the counter positions, the circle areas are proportional to the logarithms of the counter pulse heights and the circle colors denote the counter times. The point where two lines intersect shows the position of the shower core, and the arrow labeled by $\mathbf{u}$ is the shower axis projected onto the ground. b) An illustration of the SD time fit. Counter time is plotted versus distance along the u-axis (points). Solid line represents the fit expectation time for counters that lie directly on the $\mathbf{u}$-axis, dashed and dotted lines are the fit expectation times for counters that are $2 \mathrm{~km}$ and $4 \mathrm{~km}$ off the $\mathbf{u}$-axis, respectively. c) Lateral distribution fit to the modified AGASA function. Counter pulse height is plotted versus the perpendicular distance from the shower axis. Solid line represents the fit curve.

\section{Data and MC comparison}

Finally, we show a comparison between the data and the $\mathrm{MC}$ of the distributions of the geometrical variables, and the variables related to lateral profiles of the showers as shown in 
figure 5. The real data used here were collected from KM sub array in the period period between 8th Oct. 2019 and 7th Oct. 2020. The data and MC comparison is performed after the quality cuts of (i) Nstclust $\geq 4$, (ii) zenith angle $\theta \leq 55^{\circ}$, (iii) $\chi^{2} /$ ndof $\leq 4$, (iv) directional error from the fit $\left(\sigma_{\text {dir }}\right) \leq 8^{\circ}$, (v) fractional error of $\mathbf{S} 800$ from the fit $\left(\sigma_{S 800} / S 800\right) \leq 0.5$, and (vi) reconstructed core position of at least $400 \mathrm{~m}$ away from the edge of the array.

A roughly good agreement was obtained in these variables.

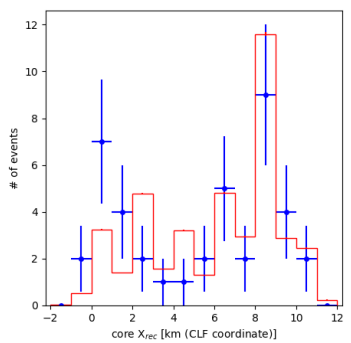

(a) Reconstructed core positions on the west-east

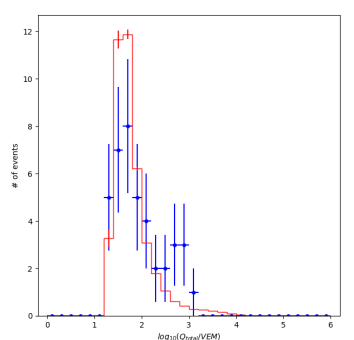

(e) The total signal per event (f) The $\chi^{2} /$ ndof for the geometrical/lateral combined fit

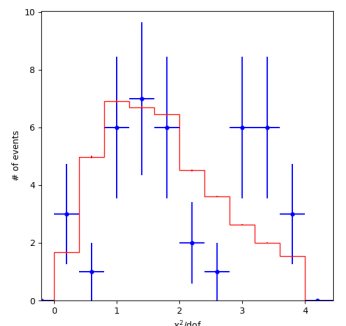

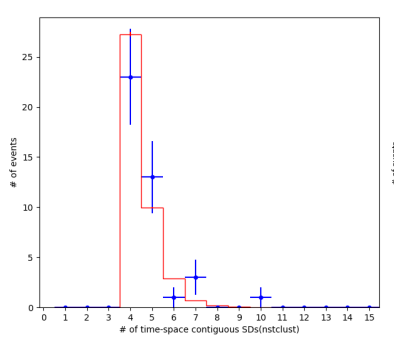

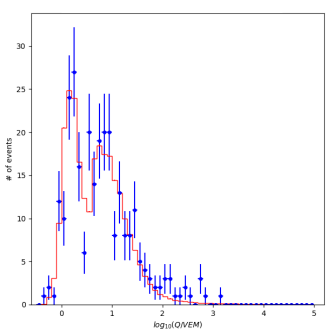

- (c) The number of spatially (d) The total signal per deand temporarily contiguous tector counters per event

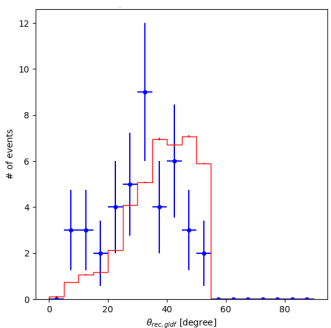

(g) Zenith angle

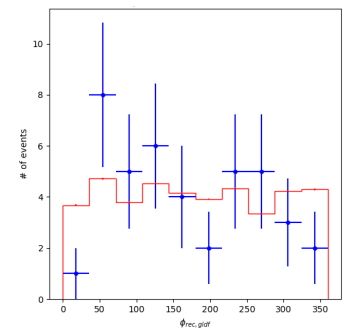

(h) Azimuth angle

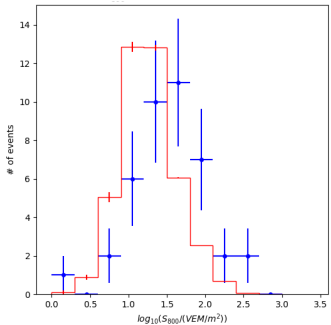

(i) $\mathrm{S} 800$

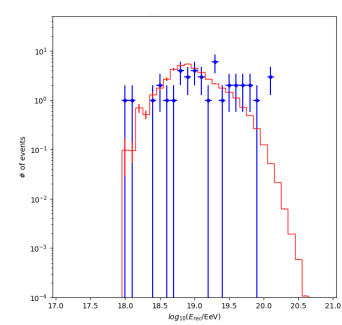

(j) Estimated energy

Figure 5: A comparison between the data and the MC of the distributions of the geometrical variables, and the variables related to lateral profiles of the showers. In each histogram, the real data are represented by blue crosses and MC simulation data are represented by solid red lines. The distributions of reconstructed core positions on the west-east (a) and south-north (b) axes. (c) The number of spatially and temporarily contiguous counters per event. The total signal per detector (d) and per event (e). (f) The $\chi^{2} /$ ndof for the geometrical/lateral combined fit. The distributions of zenith angle (g) and azimuth angle (h). The distributions of S800 (i) and estimated energy (j) values. The real data used here were collected from KM sub array in the period period between 8th Oct. 2019 and 7th Oct. 2020. 


\section{Summary}

TA found evidence of anisotropy in the distributions of arrival directions of cosmic rays with energies greater than $57 \mathrm{EeV}$. To collect data more rapidly, we planned to expand the TA SD array toward four times TA SD adding 500 SDs with 2.08-km spacing. We deployed 257 SDs in February and March of 2019.

On the basis of MC simulation, we expect 27\% energy resolution, 2.4 degree angular resolution for cosmic rays with energies greater than $57 \mathrm{EeV}$.

We compared data and MC simulation for the distributions of the geometrical variables, and the variables related to lateral profiles of the showers. A roughly good agreement was obtained in these variables.

\section{References}

[1] Kawai, H. et al. (2008). Telescope Array Experiment. Nucl. Phys. B: Proc. Suppl 175-176:221.

[2] Abbasi, R.U. et al. (2014). Indications of intermediate-scale anisotropy of cosmic rays with energy greater than $57 \mathrm{EeV}$ in the northern sky measured with the surface detector of the Telescope Array experiment. Astrophys. J. Lett., 790(2):L21.

[3] Abbasi, R.U. et al. (2018). Evidence of intermediate-scale Energy Spectrum Anisotropy of Cosmic Rays E $\geq 10^{19.2} \mathrm{eV}$ with the Telescope Array Surface Detector. Astrophys. J. 862(2):91.

[4] Abbasi, R.U. et al. (2020). Evidence for a Supergalactic Structure of Magnetic Deflection Multiplets of Ultra-high-energy Cosmic Rays. Astrophys. J. 899(1):86.

[5] Abbasi, R.U. et al. (2021). Surface detectors of the TAx4 experiment. submitted to Nucl. Instrum. Meth. A.

[6] Abu-Zayyad, T. et al. (2012). The surface detector array of the Telescope Array experiment. Nucl. Instrum. Meth. A, 689:87.

[7] Abu-Zayyad, T. et al. (2014). CORSIKA Simulation of the Telescope Array Surface Detector, arXiv:1403.0644 [astro-ph.IM].

[8] Heck, D. et al. Forsch. Karlsruhe Wiss. Ber. FZKA 6019. http://bibliothek.fzk.de/zb/berichte/FZKA6019.pdf (1998).

[9] Ostapchenko, S. (2006) QGSJETII: Towards reliable description of very high energy hadronic interactions. Nucl. Phys. Proc. Supp 151:143.

[10] Ferrari, A., Sala, P.R., Fasso, A., Ranft J. (2005). FLUKA: A multi-particle transport code (Program version 2005). Tech. Rep. 2005-010, CERN (2005).

[11] Nelson, W.R., Hirayama, H., Rogers, D.W.O. (1985). The EGS4 Code System. SLAC-265.

[12] Stokes, B.T., Ivanov, D., Matthews, J.N., Thomson, G.B. (2012). Dethinning extensive air shower simulations Astropart. Phys. 35:759.

[13] Agostinelli, S. et al. (2003). GEANT4 : A simulation toolkit. Nucl. Instrum. Meth. A506:250. 


\section{Full Authors List: Telescope Array Collaboration}

R.U. Abbasi ${ }^{1,2}$, T. Abu-Zayyad ${ }^{1,2}$, M. Allen ${ }^{2}$, Y. Arai ${ }^{3}$, R. Arimura ${ }^{3}$, E. Barcikowski ${ }^{2}$, J.W. Belz ${ }^{2}$, D.R. Bergman ${ }^{2}$, S.A. Blake², I. Buckland ${ }^{2}$, R. $\mathrm{Cady}^{2}$, B.G. Cheon ${ }^{4}$, J. Chiba ${ }^{5}$, M. Chikawa ${ }^{6}$, T. Fujii ${ }^{7}$, K. Fujisue ${ }^{6}$, K. Fujita ${ }^{3}$, R. Fujiwara ${ }^{3}$, M. Fukushima ${ }^{6}$, R. Fukushima ${ }^{3}$, G. Furlich ${ }^{2}$, R. Gonzalez ${ }^{2}$, W. Hanlon ${ }^{2}$, M. Hayashi ${ }^{8}$, N. Hayashida ${ }^{9}$, K. Hibino ${ }^{9}$, R. Higuchi ${ }^{6}$, K. Honda ${ }^{10}$, D. Ikeda ${ }^{9}$, T. Inadomi ${ }^{11}$, N. Inoue ${ }^{12}$, T. Ishii ${ }^{10}$, H. Ito $^{13}$, D. Ivanov ${ }^{2}$, H. Iwakura ${ }^{11}$, A. Iwasaki ${ }^{3}$, H.M. Jeong ${ }^{14}$, S. Jeong ${ }^{14}$, C.C.H. Jui ${ }^{2}$, K. Kadota ${ }^{15}$, F. Kakimoto ${ }^{9}$, O. Kalashev ${ }^{16}$, K. Kasahara ${ }^{17}$, S. Kasami ${ }^{18}$, H. Kawai ${ }^{19}$, S. Kawakami ${ }^{3}$, S. Kawana ${ }^{12}$, K. Kawata ${ }^{6}$, I. Kharuk ${ }^{16}$, E. Kido ${ }^{13}$, H.B. Kim ${ }^{4}$, J.H. Kim ${ }^{2}$, J.H. Kim ${ }^{2}$, M.H. Kim ${ }^{14}$, S.W. Kim ${ }^{14}$, Y. Kimura ${ }^{3}$, S. Kishigami ${ }^{3}$, Y. Kubota ${ }^{11}$, S. Kurisu ${ }^{11}$, V. Kuzmin ${ }^{16^{*}}$, M. Kuznetsov ${ }^{16,20}$, Y.J. Kwon ${ }^{21}$, K.H. Lee ${ }^{14}$, B. Lubsandorzhiev ${ }^{16}$, J.P. Lundquist ${ }^{2,22}$, K. Machida ${ }^{10}$, H. Matsumiya ${ }^{3}$, T. Matsuyama ${ }^{3}$, J.N. Matthews ${ }^{2}$, R. Mayta ${ }^{3}$,

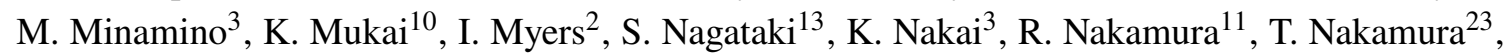
T. Nakamura ${ }^{11}$, Y. Nakamura ${ }^{11}$, A. Nakazawa ${ }^{11}$, E. Nishio ${ }^{18}$, T. Nonaka ${ }^{6}, \mathrm{H}_{\text {. Oda }}{ }^{3}, \mathrm{~S}_{\text {O Ogio }}{ }^{3,24}$, M. Ohnishi ${ }^{6}$, H. Ohoka ${ }^{6}$, Y. Oku ${ }^{18}$, T. Okuda ${ }^{25}$, Y. Omura ${ }^{3}$, M. Ono ${ }^{13}$, R. Onogi ${ }^{3}$, A. Oshima ${ }^{3}$, S. Ozawa ${ }^{26}$, I.H. Park ${ }^{14}$, M. Potts ${ }^{2}$, M.S. Pshirkov ${ }^{16,27}$, J. Remington ${ }^{2}$, D.C. Rodriguez ${ }^{2}$, G.I. Rubtsov ${ }^{16}$, D. Ryu ${ }^{28}$, H. Sagawa ${ }^{6}$, R. Sahara ${ }^{3}$, Y. Saito ${ }^{11}$, N. Sakaki ${ }^{6}$, T. Sako ${ }^{6}$, N. Sakurai ${ }^{3}$, K. Sano ${ }^{11}$, K. Sato ${ }^{3}$, T. Seki ${ }^{11}$, K. Sekino ${ }^{6}$, P.D. Shah ${ }^{2}$, Y. Shibasaki ${ }^{11}$, F. Shibata ${ }^{10}$, N. Shibata ${ }^{18}$, T. Shibata ${ }^{6}$, H. Shimodaira ${ }^{6}$, B.K. Shin ${ }^{28}$, H.S. Shin ${ }^{6}$, D. Shinto ${ }^{18}$, J.D. Smith ${ }^{2}$, P. Sokolsky ${ }^{2}$, N. Sone ${ }^{11}$, B.T. Stokes ${ }^{2}$, T.A. Stroman ${ }^{2}$, Y. Takagi ${ }^{3}$, Y. Takahashi $^{3}$, M. Takamura $^{5}$, M. Takeda $^{6}$, R. Takeishi ${ }^{6}$, A. Taketa ${ }^{29}$, M. Takita ${ }^{6}$, Y. Tameda ${ }^{18}$, H. Tanaka ${ }^{3}$, K. Tanaka ${ }^{30}$, M. Tanaka ${ }^{31}$, Y. Tanoue ${ }^{3}$, S.B. Thomas ${ }^{2}$, G.B. Thomson ${ }^{2}$, P. Tinyakov ${ }^{16,20}$ I. Tkachev $^{16}$, H. Tokuno ${ }^{32}$, T. Tomida ${ }^{11}$, S. Troitsky ${ }^{16}$, R. Tsuda ${ }^{3}$, Y. Tsunesada ${ }^{3,24}$, Y. Uchihori ${ }^{33}$, S. Udo ${ }^{9}$, T. Uehama ${ }^{11}$, F. Urban ${ }^{34}$, T. Wong ${ }^{2}$, K. Yada $^{6}$, M. Yamamoto ${ }^{11}$, K. Yamazaki ${ }^{9}$, J. Yang ${ }^{35}$, K. Yashiro ${ }^{5}$, F. Yoshida ${ }^{18}$, Y. Yoshioka ${ }^{11}$, Y. Zhezher ${ }^{6,16}$, and Z. Zundel ${ }^{2}$

${ }^{1}$ Department of Physics, Loyola University Chicago, Chicago, Illinois, USA

${ }^{2}$ High Energy Astrophysics Institute and Department of Physics and Astronomy, University of Utah, Salt Lake City, Utah, USA

${ }^{3}$ Graduate School of Science, Osaka City University, Osaka, Osaka, Japan

${ }^{4}$ Department of Physics and The Research Institute of Natural Science, Hanyang University, Seongdong-gu, Seoul, Korea

${ }^{5}$ Department of Physics, Tokyo University of Science, Noda, Chiba, Japan

${ }^{6}$ Institute for Cosmic Ray Research, University of Tokyo, Kashiwa, Chiba, Japan

7 The Hakubi Center for Advanced Research and Graduate School of Science, Kyoto University, KitashirakawaOiwakecho, Sakyo-ku, Kyoto, Japan

${ }^{8}$ Information Engineering Graduate School of Science and Technology, Shinshu University, Nagano, Nagano, Japan

${ }^{9}$ Faculty of Engineering, Kanagawa University, Yokohama, Kanagawa, Japan

${ }^{10}$ Interdisciplinary Graduate School of Medicine and Engineering, University of Yamanashi, Kofu, Yamanashi, Japan

${ }^{11}$ Academic Assembly School of Science and Technology Institute of Engineering, Shinshu University, Nagano, Nagano, Japan

${ }^{12}$ The Graduate School of Science and Engineering, Saitama University, Saitama, Saitama, Japan

${ }^{13}$ Astrophysical Big Bang Laboratory, RIKEN, Wako, Saitama, Japan

${ }^{14}$ Department of Physics, SungKyunKwan University, Jang-an-gu, Suwon, Korea

${ }^{15}$ Department of Physics, Tokyo City University, Setagaya-ku, Tokyo, Japan

${ }^{16}$ Institute for Nuclear Research of the Russian Academy of Sciences, Moscow, Russia

${ }^{17}$ Faculty of Systems Engineering and Science, Shibaura Institute of Technology, Minato-ku, Tokyo, Japan 
${ }^{18}$ Department of Engineering Science, Faculty of Engineering, Osaka Electro-Communication University, Neyagawashi, Osaka, Japan

${ }^{19}$ Department of Physics, Chiba University, Chiba, Chiba, Japan

${ }^{20}$ Service de Physique Théorique, Université Libre de Bruxelles, Brussels, Belgium

${ }^{21}$ Department of Physics, Yonsei University, Seodaemun-gu, Seoul, Korea

22 Center for Astrophysics and Cosmology, University of Nova Gorica, Nova Gorica, Slovenia

${ }^{23}$ Faculty of Science, Kochi University, Kochi, Kochi, Japan

${ }^{24}$ Nambu Yoichiro Institute of Theoretical and Experimental Physics, Osaka City University, Osaka, Osaka, Japan

${ }^{25}$ Department of Physical Sciences, Ritsumeikan University, Kusatsu, Shiga, Japan

${ }^{26}$ Quantum ICT Advanced Development Center, National Institute for Information and Communications Technology, Koganei, Tokyo, Japan

27 Sternberg Astronomical Institute, Moscow M.V. Lomonosov State University, Moscow, Russia

${ }^{28}$ Department of Physics, School of Natural Sciences, Ulsan National Institute of Science and Technology, UNIST-gil, Ulsan, Korea

${ }^{29}$ Earthquake Research Institute, University of Tokyo, Bunkyo-ku, Tokyo, Japan

${ }^{30}$ Graduate School of Information Sciences, Hiroshima City University, Hiroshima, Hiroshima, Japan

${ }^{31}$ Institute of Particle and Nuclear Studies, KEK, Tsukuba, Ibaraki, Japan

${ }^{32}$ Graduate School of Science and Engineering, Tokyo Institute of Technology, Meguro, Tokyo, Japan

33 Department of Research Planning and Promotion, Quantum Medical Science Directorate, National Institutes for Quantum and Radiological Science and Technology, Chiba, Chiba, Japan

${ }^{34}$ CEICO, Institute of Physics, Czech Academy of Sciences, Prague, Czech Republic

${ }^{35}$ Department of Physics and Institute for the Early Universe, Ewha Womans University, Seodaaemun-gu, Seoul, Korea

\section{Acknowledgements:}

The Telescope Array experiment is supported by the Japan Society for the Promotion of Science(JSPS) through Grants-in-Aid for Priority Area 431, for Specially Promoted Research JP21000002, for Scientific Research (S) JP19104006, for Specially Promoted Research JP15H05693, for Scientific Research (S) JP15H05741 and JP19H05607, for Science Research (A) JP18H03705, for Young Scientists (A) JPH26707011, and for Fostering Joint International Research (B) JP19KK0074, by the joint research program of the Institute for Cosmic Ray Research (ICRR), The University of Tokyo; by the Pioneering Program of RIKEN for the Evolution of Matter in the Universe (r-EMU); by the U.S. National Science Foundation awards PHY-1404495, PHY-1404502, PHY-1607727, PHY-1712517, PHY-1806797 and PHY-2012934; by the National Research Foundation of Korea (2017K1A4A3015188, 2020R1A2C1008230, \& 2020R1A2C2102800) ; by the Ministry of Science and Higher Education of the Russian Federation under the contract 075-15-2020-778, RFBR grant 20-02-00625a (INR), IISN project No. 4.4501.18, and Belgian Science Policy under IUAP VII/37 (ULB). This work was partially supported by the grants of The joint research program of the Institute for Space-Earth Environmental Research, Nagoya University and Inter-University Research Program of the Institute for Cosmic Ray Research of University of Tokyo. The foundations of Dr. Ezekiel R. and Edna Wattis Dumke, Willard L. Eccles, and George S. and Dolores Doré Eccles all helped with generous donations. The State of Utah supported the project through its Economic Development Board, and the University of Utah through the Office of the Vice President for Research. The experimental site became available through the cooperation of the Utah School and Institutional Trust Lands Administration (SITLA), U.S. Bureau of Land Management (BLM),

\footnotetext{
* Deceased
} 
and the U.S. Air Force. We appreciate the assistance of the State of Utah and Fillmore offices of the BLM in crafting the Plan of Development for the site. Patrick A. Shea assisted the collaboration with valuable advice and supported the collaboration's efforts. The people and the officials of Millard County, Utah have been a source of steadfast and warm support for our work which we greatly appreciate. We are indebted to the Millard County Road Department for their efforts to maintain and clear the roads which get us to our sites. We gratefully acknowledge the contribution from the technical staffs of our home institutions. An allocation of computer time from the Center for High Performance Computing at the University of Utah is gratefully acknowledged. 